\title{
Non Alcoholic Fatty Liver Disease (NAFLD) and Type 2 Diabetes Mellitus
}

\author{
Bickram Pradhan, Denis Peeyush \\ Department of Gastroentology and Hepatology, B.P.Koirala Institute of Health Sciences, Dharan, Nepal.
}

\begin{abstract}
Background: The pandemic of obesity and type2-diabetes mellitus has led to a increasing prevalence of Non Alcoholic Fatty Liver Disease (NAFLD) globally, including developing countries. The current epidemic of NAFLD is reshaping the field of hepatology because patients with NAFLD are at increased risk for not only liver-related morbidity and mortality but also cardiovascular disease. NAFLD also increases the risk of developing diabetes. Hence patients with diabetes need to be screened for the presence of NAFLD and vice- versa. It is of paramount importance to differentiate between simple steatosis from Non Alcoholic Steatohepatitis (NASH), the later being more associated with hepatic as well as extra hepatic complications.
\end{abstract}

Key Words: Diabetes Mellitus; Liver Injury; NAFLD

Abbreviations: NAFLD= Non-Alcoholic Fatty Liver Disease; NASH= Non Alcoholic Steatohepatitis; $\mathrm{HCC}=$ Hepatocellular carcinoma;EASL $=$ European Association for the Study of the Liver);AASLD = American Association for the Study of Liver Diseases;EASD = The European Association for the Study of Diabetes);EASO=European Association for the Study of Obesity ; CCL2-CCL5 = C-C chemokine receptor types 2 / type 5; FXR = Farnesoid $\mathrm{X}$ receptor

\section{INTRODUCTION}

NAFLD is a broad spectrum of diseases consisting of patients with simple steatosis or Nonalcoholic fatty liver, Non alcoholic steato hepatitis (NASH), NASH-related cirrhosis, and NASH-related HCC. In NAFL there is $\geq 5 \%$ hepatic steatosis without significant inflammation. NASH is defined as steatosis and inflammation associated with the presence of one of the three additional features: ballooning of hepatocytes, Mallory hyaline, and fibrosis on liver histology. NASH is usually a histological diagnosis.

It is very important to differentiate between NAFLD and NASH to determine the prognosis, risk of progression, and for assessing the liver-related and cardiovascular morbidity and mortality, which occurs more frequently in patients with NASH as

Corresponding author:

Prof Dr. Bickram Pradhan, Professor and Head,

Department of Gastroenterology and Hepatology, BP Koirala Institute of Health Sciences, Dharan, Nepal,

Email: bikram.p@gmail.com compared to simple steatosis.

Why should NAFLD be of interest to diabetologists?

NAFLD is strongly associated with type 2 diabetes mellitus and abdominal obesity.

NAFLD is the hepatic component of metabolic syndrome. The prevalence of NAFLD in diabetes mellitus has been reported to be $74 \%{ }^{1}, 57 \%^{2}$ and $70 \%{ }^{3}$ in different studies. As lifestyles have become increasingly sedentary with obesity and type 2 diabetes mellitus pandemic, NAFLD is rapidly becoming the leading cause of chronic liver disease worldwide ${ }^{4}$. It is projected to be the principal etiology for liver transplantation within the next decade.

NAFLD is a risk factor for Type 2 Diabetes mellitus and cardiovascular disease

NAFLD is associated not only with liver-related morbidity and mortality, but also with an increased risk of developing both cardiovascular disease and type 2 diabetes mellitus ${ }^{5}$. 
Non Alcoholic Fatty Liver Disease (NAFLD) ...

Jour of Diab and Endo Assoc of Nepal 2019; 3 (2): (53-59)

ISSN Print 2594-3367 ISSN Online 2631-2107

NAFLD was a significant predictor for future diabetes in a Japanese middle-aged health check population, especially in women. The relative risk of diabetes associated with fatty liver was 4.8 [95\% confidence interval (CI) $3.0-7.8, \mathrm{p}<0.0001]$ in men and 14.5 (95\% CI 7.0-30.1, p<0.0001) in women ${ }^{6}$. Current evidence shows that with resolution of fatty liver, there is a potential for decreasing risk of incident type 2 diabetes mellitus ${ }^{7}$.

NAFLD is also significantly associated with a moderately increased cardiovascular disease risk among type 2 diabetic patients This risk is independent of other classical risk factors and only party explained by the presence of metabolic syndrome (8). In fact, patients with NAFLD are twice as likely to die of cardiovascular disease than liver disease and liver disease is only the third leading cause of death in patients with NAFLD, following cardiovascular disease and malignancy ${ }^{9}$.

\section{Type 2 Diabetes increases the risk of NAFLD} progressions to more advanced liver disease

Type 2 diabetes mellitus is one of the strongest clinical predictors of the progression of NAFLD to NASH and cirrhosis (10). The progression of NAFLD to NASH in non-diabetic individuals occurs in about $10-20 \%$ of NAFLD patients ${ }^{11}$. But the presence of Type 2 diabetes increases this risk of the progression by two- to three-fold ${ }^{12}$. The presence of diabetes also increased the risk for cirrhosis or hepatocellular carcinoma (HCC) among patients with NAFLD/NASH. In fact Type 2 diabetes has emerged as a significant predictor of worse outcomes in patients with NAFLD/NASH ${ }^{13}$. There are data to demonstrate that HCC may occur in NAFLD patients without cirrhosis ${ }^{14,15}$. These data emphasize the need to effectively diagnose NAFLD and early HCC in patients with obesity, metabolic syndrome and type 2 diabetes mellitus

\section{Diagnosis of NAFLD}

The diagnostic criteria of NAFLD includes hepatic steatosis by either imaging or histology, no other causes of steatosis and no significant alcohol consumption.$^{16}$

All individuals with metabolic risk factors should be screened with ultrasonography of the liver to identify liver fat and assessment of liver enzymes. However, ultrasonography is a relatively insensitive technique for detecting liver fat. The fatty infiltration must be at least $20-30 \%$, before ultrasonography will be able to diagnose hepatic steatosis. As an alternative, a simpler surrogate markers for diagnosing liver fat, such as the fatty liver index (a composite score derived from BMI, waist circumference, fasting triacylglycerol and $\gamma$-glutamyltransferase [GGT] concentrations) can be used as a first-line approach that also has an acceptable sensitivity and specificity for identifying liver fat ${ }^{17}$.

For diagnosis of NAFLD, other causes of steaosis should be excluded including but not limited to increased alcohol consumption, viral hepatitis, surgical procedures, use of medications and total parenteral nutrition. There is a disagreement among different guidelines in defining the threshold for alcohol intake. According to EASL ${ }^{17}$, significant alcohol consumption is defined as $>30 \mathrm{~g} / \mathrm{d}$ in men and $>20 \mathrm{~g} / \mathrm{d}$ in women. The AASLD guidance considers ${ }^{18}>21$ standard drink per week in men and $>14$ in women as significant. Asia-Pacific Guidelines ${ }^{19}$ defines significant alcohol intake as $>$ 7 standard alcoholic drinks/week (70 g ethanol) in women and $>14(140 \mathrm{~g})$ in men.

Components of the metabolic syndrome and diabetes should be screened. After making a diagnosis of NAFLD, the next step is assessment of fibrosis as the severity of fibrosis is the strongest predictor of liver-related outcome

Although histological examination of the liver is the 'gold standard' to stage NAFLD severity, it is not a feasible option due to invasiveness of the test, complications, cost involved, and poor acceptability of the patients. Hence the use of non-invasive test for detection of liver fibrosis is recommended which includes NAFLD fibrosis score, Enhanced 
Non Alcoholic Fatty Liver Disease (NAFLD) ...

Jour of Diab and Endo Assoc of Nepal 2019; 3 (2): (53-59)

ISSN Print 2594-3367 ISSN Online 2631-2107

\section{Liver Fibrosis [ELF] or FIB-4 scores.}

Depending on the results of these tests either followup at 3-5 years or specialist referral for a decision as to whether to undertake a liver biopsy, and/or initiation of therapy. Alternatively, for NAFLD patients with mild abnormalities of non-invasive fibrosis markers, further follow-up at 2 years with repeat testing is advocated ${ }^{20}$. The EASL-EASDEASO guidelines recommend that all patients with elevated LFTs because of NAFLD and advanced fibrosis should be referred to a hepatologist ${ }^{20}$.

\section{Role of liver biopsy}

Liver biopsy should be reserved for the following conditions. NAFLD patients who are at increased risk of having steato hepatitis and/or advanced fibrosis and in patients with suspected NAFLD in whom competing etiologies for hepatic steatosis and the presence and/or severity of coexisting CLDs cannot be excluded without a liver biopsy ${ }^{18}$.

\section{Treatment}

Lifestyle changes: Lifestyle modification including diet, exercise, and weight loss has been recommended for treatment of patients with
NAFLD. The recommendation is to give 500-1000 kcal energy deficit diet to induce a weight loss of $500-1000 \mathrm{~g} /$ week with a 7\%-10\% total weight loss. Dietary recommendation also involves exclusion of NAFLD-promoting components (processed food, and food and beverages high in added fructose. Weight loss has been reported to be associated with improvement in histologic features. 150-200 $\mathrm{min} /$ week of moderate intensity aerobic physical activities in 3-5 sessions are generally preferred (brisk walking, stationery cycling) ${ }^{17}$. However, lifestyle modification is difficult to achieve and to sustain

\section{Pharmacological treatment Whom to treat?}

Drug therapy is indicated for progressive NASH (bridging fibrosis and cirrhosis) and early-stage NASH with increased risk of fibrosis progression (age >50 years; diabetes, MetS, increased ALT or active NASH with high necroinflammatory activity ${ }^{17}$.

The recommendation of drug therapy for the treatment of NAFLD by different society are summarized in table 1.

Table 1.

\begin{tabular}{|l|l|l|l|}
\hline Drugs & EASL & ASIA-PACIFIC & AASLD \\
\hline Metformin & Insufficient evidence & Not beneficial & Not beneficial \\
\hline Vitamin E & Insufficient evidence & Not beneficial & Consider use in \\
\hline & & & non-diabetic, biopsy- \\
\hline & & & proven NASH \\
\hline PPAR-gamma agonists & Consider use in selected & Insufficient evidence & Pioglitazone indicated \\
\hline & diabetic patients & in Asian & in biopsy-proven \\
\hline & & & NASH (regardless of \\
\hline & & & diabetes) \\
\hline UDCA & Not beneficial & Not mentioned & Not beneficial \\
\hline Silymarin & Not mentioned & Insufficient evidence, & Not mentioned \\
\hline & & potentially useful & \\
\hline Statins & Safe but not beneficial & Safe but not beneficial & Safe but not beneficial \\
\hline
\end{tabular}


Non Alcoholic Fatty Liver Disease (NAFLD) ...

Jour of Diab and Endo Assoc of Nepal 2019; 3 (2): (53-59)

ISSN Print 2594-3367 ISSN Online 2631-2107

\section{Future pharmacological options of NASH} Gut microbiome

NAFLD is associated with increased gut permeability and transportation of gut metabolites and bacterial products into the portal circulation.

An increased lipopolysaccharide levels in the circulation occurs, which binds to the monocyte differentiation antigen (CD14)-TLR-4 complex triggering an inflammatory reaction and insulin resistance. The gut microbiota is also involved in choline metabolism by converting it into toxic dimethylamine and trimethylamine, which are transported to liver and converted into trimethylamine oxide (TMAO) that causes liver inflammation and damage ${ }^{21}$.

Changing the gut mocrobiota may be a treatment option in NAFLD. Solithromycin, a macrolide antibiotic with anti-inflammatory properties, was found to improve NASH in animal studies and is currently being studied in a phase 2 clinical trial ${ }^{22}$.

\section{Antiobesity medications}

Orlistat is a gut lipase inhibitor which decreases the absorption of dietary fats. A small pilot study demonstrated reduction in hepatic steatosis associated with Orlistat-induced weight $\operatorname{loss}^{23}$. However, it is not currently recommended as a treatment for NAFLD, but can be prescribed as an adjunct medication to help with weight loss in the NAFLD patients ${ }^{24}$.

\section{Peroxisome proliferator-activator receptors (PPARs)}

PPARs are nuclear receptors that bind fatty acids and fatty acid derivatives to regulate a number of metabolic processes. The three PPAR agonist considered for use in NAFLD are Elafibranor (dual PPAR $\alpha / \delta$ agonist) Pioglitazone (PPAR $\gamma$ agonist), and Saroglitazar (dual PPAR $\alpha / \gamma$ agonist).

Elafibranor has been shown to improve peripheral tissue insulin sensitivity and reduce alanine aminotransferase (ALT) levels in patients with metabolic syndrome ${ }^{25}$. A phase 3 trial using elafibrinor versus placebo for 72 weeks is currently ongoing for the treatment of NASH. (NCT02704403).

PPAR $\gamma$ agonists like thiazolidinediones are used in the treatment of diabetes and demonstrated to be effective in NASH ${ }^{26}$. However, undesirable side effects and the possible need for long-term therapy have limited widespread acceptance.

Saroglitazar is a dual PPAR $\alpha / \gamma$ agonists which combines the beneficial effects of activating both PPAR receptors. It has been shown to improve diabetic dyslipidemia ${ }^{27}$ and is currently approved in India for this indication. A retrospective study of NAFLD patients with dyslipidemia treated with saroglitazar for 24 weeks demonstrated a significant decrease in ALT compared with baseline ${ }^{28}$. A phase 3 trial is currently ongoing in India to assess the effect of saroglitazar versus placebo for 52 weeks in biopsy proven noncirrhotic NASH (Clinical Trials Registry-India CTRI/2015/10/006236).

\section{Farnesoid X receptor agonist}

Bile acids can negatively regulate bile acid synthesis, decrease hepatic gluconeogenesis, and lipogenesis through interaction with their intracellular receptor, the farnesoid X receptor (FXR).

A synthetic bile acid agonist of FXR, obeticholic acid (OCA) was evaluated in a phase $2 b$ clinical trial (FLINT) which included biopsy-proven noncirrhotic NASH patients who were randomized to OCA $25 \mathrm{mg}$ /day versus placebo for 72 weeks ${ }^{29}$. This important study established the role of FXR in NASH by showing that the FXR bile acid agonist OCA improved histological features of NASH. Histological improvement, with no worsening of fibrosis was demonstrated in a significant study participants on OCA as compared to placebo ( $45 \%$ vs $21 \%, \mathrm{P}=0.0002)$. and decrease in fibrosis score was also significant. A phase 3 trial to compare the effectiveness of OCA versus placebo for noncirrhotic biopsy-proven NASH is currently ongoing (NCT02548351). 
Non Alcoholic Fatty Liver Disease (NAFLD) ...

Jour of Diab and Endo Assoc of Nepal 2019; 3 (2): (53-59)

ISSN Print 2594-3367 ISSN Online 2631-2107

Incretins, dipeptidyl peptidase-4 inhibitors and sodium-glucose cotransporter 2 inhibitors.

Glucagon-like peptide GLP-1 receptor agonists or incretin mimetics, liraglutide was investigated in a phase 2 trial for its effectiveness in biopsy proven $\mathrm{NASH}^{30}$. This important study (LEAN trial) established the utility of GLP-1 pathway in NASH by demonstrating that the long-acting GLP-1 analogue, liraglutide, led to histological resolution of NASH. The primary end point of histological resolution of NASH without worsening of fibrosis was reached by $39 \%$ of study participants on liraglutide versus $9 \%$ on placebo $(\mathrm{P}=0.02)$.

\section{Cenicriviroc}

Cenicriviroc is an an oral antagonist of the CCL2CCL5 receptor, A phase $2 \mathrm{~b}$ trial (CENTAUR) is investigating the effect of 2 years of cenicriviro or placebo on noncirrhotic NASH and liver fibrosis in patients with T2DM or metabolic syndrome (NCT02217475). Interim analysis at year 1 of the CENTAUR study, showed significant improvement in fibrosis and no worsening of stetaohepatitis as compared to placebo ${ }^{31}$.

\section{CONCLUSION}

NASH is become a leading cause for chronic liver disease worldwide due to the pandemic of diabetes mellitus and obesity. NAFLD and especially NASH also confer an independent risk of adverse cardiovascular events in affected individuals beyond that conferred by the shared risk factors. Hence differentiation of simple steatosis from NASH is of paramount importance along with the staging of fibrosis of liver.

Currently, a number of drugs are undergoing pivotal trails as potential therapy for NASH. The first effective drug to be approved for treatment of NASH is anticipated to be available by 2020 .

\section{References}

1. Williams CD, Stengel J, Asike MI et al (2011) Prevalence of nonalcoholic fatty liver disease and nonalcoholic steatohepatitis among a largely middle-aged population utilizing ultrasound and liver biopsy: a prospective study. Gastroenterology 140:124-131.

2. Williamson RM, Price JF, Glancy S et al (2011) Prevalence of and risk factors for hepatic steatosis and nonalcoholic Fatty liver disease in people with type 2 diabetes: the Edinburgh Type 2 Diabetes Study. Diabetes Care 34:11391144.

3. Targher G, Bertolini L, Padovani R et al (2007) Prevalence of nonalcoholic fatty liver disease and its association with cardiovascular disease among type 2 diabetic patients. Diabetes Care 30:1212-1218.

4. Weston SR, Leyden W, Murphy Ret al. (2005). Racial and ethnic distribution of nonalcoholic fatty liver in persons with newly diagnosed chronic liver disease Hepatology; Feb 4(12) 372-379.

5. Anstee QM, Targher, G., Day CP. (2013). Progression of NAFLD to diabetes mellitus, cardiovascular disease or cirrhosis. Nature Reviews Gastroenterology \& Hepatology; 10 (6), 330-344.

6. Tokita Y, Maejima Y, Shimomura K, et al. (2017). Non-alcoholic Fatty Liver Disease Is a Risk Factor for Type 2 Diabetes in Middleaged Japanese Men and Women. Intern Med.; 56(7): 763-771.

7. Sung KC, Wild SH, Byrne CD (2013) Resolution of fatty liver and risk of incident diabetes. J Clin Endocrinol Metab 98:36373643.

8. Targher G, Bertolini L, Poli F., et al (2005). Nonalcoholic fatty liver disease and risk of future cardiovascular events among type 2 diabetic patients. Diabetes.Dec;54(12):3541-6.

9. Friedman SL, Neuschwander-Tetri BA, Rinella M., et al. Mechanisms of NAFLD development and therapeutic strategies. Nat Med. (2018); 24(7): 908-922. 
10. Adams L. A., Lymp J. F., St Sauver J., Sanderson S. O., Lindor K. D., Feldstein A., et al. (2005). The natural history of nonalcoholic fatty liver disease: a population-based cohort study. Gastroenterology. 129 (1), 113-121.

11. Lazo M., Clark J. M. (2008). The epidemiology of nonalcoholic fatty liver disease: a global perspective. Semin Liver Dis. 28, 339-350.

12. Portillo-Sanchez P., Bril F., Maximos M., Lomonaco R., Biemacki D., Orsak B., et al. (2015). High Prevalence of Nonalcoholic Fatty Liver Disease in Patients With Type 2 Diabetes Mellitus and Normal Plasma Aminotransferase Levels. J. Clin. Endocrinol Metab. 100 (6), 2231-2238.

13. Alexander M, Loomis AK, van der lel j., et al (2019). Risks and clinical predictors of cirrhosis and hepatocellularcarcinoma diagnoses in adults with diagnosed NAFLD: real-worldstudy of 18 million patients in four European cohorts. BMC Med.May 20; 17(1): 95.

14. Dyson J, Jaques B, Chattopadyhay D et al (2014) Hepatocellular cancer: the impact of obesity, type 2 diabetes and a multidisciplinary team. J Hepatol 60:110-117.

15. Perumpail RB, Wong RJ, Ahmed A, Harrison SA (2015) Hepatocellular carcinoma in the setting of non-cirrhotic nonalcoholic fatty liver disease and the metabolic syndrome: US experience. Dig Dis Sci 60:3142-3148.

16. Leoni S, Tovoli F Napoli L., et al . Current guidelines for the management of non-alcoholic fatty liver disease (2018). A systematic review with comparative analysis. World $\mathrm{J}$ Gastroenterol. Aug 14;24(30):3361-3373.

17. European Association for the Study of the Liver (EASL) European Association for the Study of Diabetes (EASD); European Association for the Study of Obesity (EASO). EASLEASD-EASO Clinical Practice Guidelines for the management of non-alcoholic fatty liver disease. (2016). J. Hepatol.64: 1388-1402.

18. Chalasani N, Younossi Z, Lavine JE., et al.(2018) The diagnosis and management of nonalcoholic fatty liver disease: Practice guidance from the American Association for the Study of Liver Diseases. Hepatology.67; 328-357.

19. Wong VW, Chan WK, Chitturi S, Chawla Y, Dan YY, Duseja A, Fan J, Goh KL, Hamaguchi M, Hashimoto E, et al. (2018).Asia-Pacific Working Party on Non-alcoholic Fatty Liver Disease guidelines 2017-Part 1: Definition, risk factors and assessment. J Gastroenterol Hepatol; 33:70-85.

20. ByrneCD, Targher G. EASL-EASD. EASO Clinical Practice Guidelines for the management of non-alcoholic fatty liver disease: is universal screening appropriate? (2016).Diabetologia. Jun; 59(6): 1141-4.

21. Wang Z., Klipfell E., Bennett B.J., Koeth R., Levison B.S., Dugar B., Feldstein A.E., Britt E.B., Fu X., Chung Y.M., et al.(2011). Gut flora metabolism of phosphatidylcholine promotes cardiovascular disease. Nature.472: 57-63.

22. Fernandes P (2015). Mechanism of anti-NASH effects of Solithromycin in a predictive NASH HCC mouse model. Hepatology.; 62:1301A-A

23. Harrison SA, Fecht W, Brunt EM, et al. (2009). Orlistat for overweight subjects with nonalcoholic steatohepatitis: a randomized, prospective trial. Hepatology.; 49:80-86.

24. Banini BA, Sanyal AJ. (2017). Current and future pharmacologic treatment of nonalcoholic steatohepatitis. Curr Opin Gastroenterol. May; 33(3): 134-141.

25. Cariou B, Hanf R, Lambert-Porcheron S, et al. (2013). Dual peroxisome proliferator-activated receptor alpha/delta agonist GFT505 improves hepatic and peripheral insulin sensitivity in abdominally obese subjects. Diabetes Care.36: 2923-2930.

26. Boettcher E, Csako G, Pucino F, et al.(2012) Meta-analysis: pioglitazone improves liver histology and fibrosis in patients with nonalcoholic steatohepatitis. Aliment Pharmacol Ther.35: 66-75.

27. Jani RH, Pai V, Jha P, et al. (2014).A multicenter, prospective, randomized, double- 
blind study to evaluate the safety and efficacy of Saroglitazar 2 and $4 \mathrm{mg}$ compared with placebo in type 2 diabetes mellitus patients having hypertriglyceridemia not controlled with atorvastatin therapy (PRESS VI) Diabetes Technol Ther.16:63-71.

28. Saboo B, Prajapati A, Joshi S. (2015). To Assess the Effect of $4 \mathrm{mg}$ Saroglitazar on patients of diabetes dyslipidemia with nonalcoholic fatty liver disease. Diabetes. 64:A180-A.

29. Neuschwander-Tetri BA, Loomba R, Sanyal AJ, et al., (2015). Farnesoid X nuclear receptor ligand obeticholic acid for noncirrhotic, nonalcoholic steatohepatitis (FLINT): a multicentre, randomised, placebo-controlled trial. Lancet. 385:956-965.
30. Armstrong MJ, Gaunt P, Aithal GP, et al.(2016). Liraglutide safety and efficacy in patients with nonalcoholic steatohepatitis (LEAN): a multicentre, double-blind, randomised, placebo-controlled phase 2 study. Lancet.387: 679-690.

31. Friedman S, Sanyal A, Goodman Z, et al. (2016). Efficacy and safety study of cenicriviroc for the treatment of nonalcoholic steatohepatitis in adult subjects with liver fibrosis: CENTAUR Phase $2 \mathrm{~b}$ study design. Contemp Clin Trials. 47:356-365. 